\title{
Predictors of achieving remission in schizophrenia patients treated with paliperidone palmitate 3-month formulation
}

This article was published in the following Dove Medical Press journal: Neuropsychiatric Disease and Treatment

\author{
Abigail I Nash' \\ Ibrahim Turkoz ${ }^{2}$ \\ Adam J Savitz ${ }^{2}$ \\ Maju Mathews ${ }^{2}$ \\ Edward Kim' \\ 'Janssen Scientific Affairs, LLC, \\ Titusville, NJ 08560, USA; ${ }^{2}$ Janssen \\ Research and Development, LLC, \\ Titusville, NJ 08560, USA
}

Correspondence: Edward Kim Janssen Scientific Affairs, LLC,

I 25 Trenton-Harbourton Road,

Titusville, NJ 08560, USA

Tel +l 6097304345

Email ekim37@its.jnj.com
Purpose: Long-acting injectable (LAI) antipsychotic paliperidone palmitate 3-month formulation (PP3M) is indicated in the United States for the treatment of schizophrenia only after adequate treatment with paliperidone palmitate 1-month formulation (PP1M) for $\geq 4$ months. This analysis aimed to identify patient and disease characteristics during PP1M treatment associated with greater likelihood of achieving remission after transition to PP3M.

Methods: A post hoc analysis of a randomized, Phase III, double-blind, noninferiority trial of PP3M vs PP1M (ClinicalTrials.gov identifier: NCT01515423) was conducted in adult patients with schizophrenia. Patients achieving clinical stability after 17 weeks of open-label PP1M were randomized to 48 weeks of double-blind treatment with PP3M or PP1M. The primary objective of this exploratory post hoc analysis was to identify demographic and/or clinical variables associated with persistent remission after treatment with PP3M. Multiple logistic regression analysis identified the following significant predictors of remission: Positive and Negative Syndrome Scale (PANSS) Marder negative symptom factor score, Clinical Global Impression-Severity (CGI-S) total score, and Personal and Social Performance (PSP) total score.

Results: At double-blind baseline, a 1-point reduction in Marder negative symptom factor score was associated with a $20 \%$ increase in the odds of achieving remission after PP $3 \mathrm{M}$ treatment; 1-point reduction in CGI-S was associated with a doubling in remission odds; and 7- and 10-point improvements in PSP scores, respectively, were associated with $42 \%$ and $65 \%$ increases in remission odds.

Conclusion: Patients with early clinically meaningful improvements in disease symptoms and severity while establishing stable PP1M dosage are more likely to achieve remission after transition to PP3M.

Keywords: long-acting injectable, symptomatic remission, double-blind treatment

\section{Introduction}

Schizophrenia is characterized by negative and psychotic symptoms, cognitive deficits, psychosocial impairment, and diminished quality of life, ${ }^{1,2}$ resulting in heavy humanistic and economic burdens on patients, caregivers, health care providers, and society. ${ }^{2-6}$ Symptom remission is a necessary step toward functional remission and recovery, ${ }^{7}$ which entails being able to maintain meaningful relationships and functioning socially and vocationally within the community. ${ }^{7,8}$ According to the 2005 consensus definition of the Remission in Schizophrenia Working Group (RSWG), ${ }^{7}$ remission is "a state in which patients have experienced an improvement in core signs and symptoms to the extent that any remaining symptoms are of such low intensity that they no longer interfere significantly with behavior and are below the threshold typically utilized in 
justifying an initial diagnosis of schizophrenia." "7 The RSWG developed operational criteria to assess patients for remission and, given the long-term course and intrinsic characteristics of schizophrenia, specified 6 months as the minimum period during which symptomatic improvement must be maintained. ${ }^{7,9}$ Analyses assessing the relationship between RSWG remission status and other outcome dimensions have shown that patients in remission often have better overall symptomatic status and level of function and, to a lesser extent, better cognitive performance and quality of life. . $^{10,11}$

For patients with schizophrenia, continuous treatment coverage with antipsychotic medications plays an important role in achieving and maintaining symptom control. ${ }^{12}$ Uncontrolled symptoms often lead to disruptions in functioning and productivity, increased risk of hospitalization, and increased caregiver burden. ${ }^{13,14}$ Interruptions in medication coverage with oral antipsychotic medications are common in this patient population. ${ }^{15-17}$

Long-acting injectable (LAI) antipsychotics eliminate the need for patients with schizophrenia to remember to take daily oral antipsychotics ${ }^{18}$ and may potentially help reduce relapse frequency. ${ }^{18}$ Due to their extended half-lives, LAIs may represent a buffer against medication interruptions, providing clinicians and caregivers with an extended opportunity to ensure continued follow-up and treatment continuity. ${ }^{19}$ Because LAIs are administered by a health care provider, recognition of nonadherence is easier compared with selfadministered oral antipsychotics. ${ }^{20}$

Paliperidone palmitate 3-month formulation (PP3M) is an LAI administered once every 3 months for the treatment of schizophrenia. ${ }^{21}$ Its efficacy and safety in patients with schizophrenia were evaluated in 2 Phase III, randomized, controlled trials. ${ }^{22,23}$ In the first study, patients symptomatically stable after a total of 7 months of treatment (4 months of treatment with paliperidone palmitate 1-month formulation $[\mathrm{PP} 1 \mathrm{M}]$ and a single dose of PP3M) and subsequently randomized to PP3M demonstrated a significantly delayed median time to relapse (not estimable) compared with those randomized to placebo (274 days); $93 \%$ of the subjects receiving PP3M had not experienced relapse at the time of the interim analysis. ${ }^{22}$ In the second study, PP3M was noninferior when compared with PP1M. ${ }^{23}$ After 48 weeks of double-blind treatment with PP3M or PP1M, relapse rates were $8 \%$ and $9 \%$, respectively; additionally, improvements in symptoms and personal and social functioning were similar for both groups. ${ }^{23}$ Symptomatic remission ${ }^{7}$ was achieved by $>50 \%$ of the patients in both groups during the last 6 months of the trial..$^{24}$
As per the US prescribing information, PP3M is indicated for the treatment of schizophrenia after patients have been "adequately treated" with PP1M for $\geq 4$ months; to establish a consistent maintenance dose, the last 2 doses of PP1M should be the same dosage. ${ }^{21}$ Clinical stability on PP1M in the transitional phases of the PP3M Phase III trials was defined based on rating scales (eg, on the Positive and Negative Syndrome Scale [PANSS] and Clinical Global Impression-Severity [CGI-S]). However, in clinical practice, formal rating scales are not routinely used, and "adequate treatment" on PP1M prior to the transition to PP3M has not been clearly defined, leaving clinicians without specific guidance. The aim of this exploratory post hoc analysis of the Phase III PP3M noninferiority trial ${ }^{23}$ was to identify patient or disease characteristics observed during the PP1M treatment stabilization phase associated with a greater likelihood of achieving remission following transition to PP3M in the double-blind randomized treatment phase.

\section{Methods}

\section{Study design}

Study 3011 was a randomized, Phase III, double-blind, noninferiority trial of PP3M vs PP1M (ClinicalTrials.gov identifier: NCT01515423); full details of the methodology have been reported previously. ${ }^{23}$ The study protocol and amendments were reviewed by an Independent Ethics Committee or Institutional Review Board (IEC/IRB) at each site (Table S1 for a listing of all approving IECs/ IRBs from sites with patient data included in this post hoc analysis), and the study was conducted in compliance with the Declaration of Helsinki. Written informed consent was obtained from all patients after study procedures and possible side effects were fully explained. Male and female patients aged 18-70 years had a diagnosis of schizophrenia according to the Diagnostic and Statistical Manual of Mental Disorders, Fourth Edition (DSM-IV) and a total PANSS score of 70-120 at screening and baseline, with worsening psychotic symptoms. Patients with known poor response or intolerability to paliperidone or risperidone were excluded.

All psychotropic medications (including mood stabilizers, and all oral antipsychotics, including paliperidone extended release) were discontinued prior to starting PP1M. Patients not previously treated with paliperidone received doses of paliperidone extended release $6 \mathrm{mg}$ /day for 4-6 days to establish tolerability. Open-label PP1M was then administered to all patients for 17 weeks (Day 1, $234 \mathrm{mg}$; Day 8, $156 \mathrm{mg}$; and then flexibly dosed 78, 117, 156, or $234 \mathrm{mg}$ at 
Weeks 5 and 9). The dose at Week 13 had to be the same as the dose at Week 9.

Patients achieving clinical stability (PANSS total score <70; PANSS item P1, P2, P3, P6, P7, G8, and G14 scores $\leq 4$; reduction in CGI-S score $\geq 1$ from baseline) at Weeks 14 and 17 were randomized (1:1) to a 48-week double-blind treatment period of fixed doses of PP3M (273, $410,546$, and $819 \mathrm{mg})$ or $\operatorname{PP} 1 \mathrm{M}(78,117,156$, and $234 \mathrm{mg})$. Blinding was maintained with the administration of matched monthly placebo injections in the PP3M group when not receiving active medication.

\section{Efficacy endpoints}

Efficacy endpoints in this post hoc analysis included PANSS total score, PANSS Marder factor scores (positive symptoms, negative symptoms, disorganized thoughts, uncontrolled hostility/excitement, and anxiety/depression), ${ }^{25}$ CGI-S score, and Personal and Social Performance (PSP) scores. Symptomatic remission was assessed according to RSWG criteria (score $\leq 3$ on the following PANSS symptom score items: P1 [delusions], P2 [conceptual disorganization], P3 [hallucinatory behavior], N1 [blunted affect], N4 [social withdrawal], N6 [lack of spontaneity], G5 [mannerisms/ posturing], and G9 [unusual thought content] for the last 6 months of double-blind treatment before end of study). ${ }^{7}$

\section{Statistical analyses}

All analyses were conducted on the intent-to-treat (ITT) population, defined as all randomized patients who received $\geq 1$ dose of PP3M and remained in the study for $\geq 6$ months of the double-blind phase. Demographic and clinical characteristics were compared between patients who achieved remission and those who did not use 2-sided $t$-tests for continuous endpoints and chi-square tests for categorical endpoints. Univariate logistic regression models identified demographic and/or clinical variables associated with persistent remission; significance was defined as $P<0.05$, and trend was defined as $P<0.10$.

Although univariate regression analysis is useful for showing basic relationships with achieving remission, clinical practice suggests that multiple factors can influence achievement of remission. Therefore, stepwise multiple logistic regression models were used to examine the results of the initial univariate models. Predictors were entered sequentially, and after entering each individual variable in the model, those that became nonsignificant were checked and removed from the model (entry, $P \leq 0.2$; retained, $P \leq 0.2$ ). ORs and $95 \%$ CIs were calculated. Finally, a multiple logistic regression model was fit with significant predictors identified from the stepwise model. Model fits and diagnostics were examined for violation of the assumptions of the logistic regression models and for influential data points and nonlinearity. No adjustments were made for multiplicity.

\section{Results \\ Patients}

A total of 1,429 patients were enrolled in the open-label phase; 1,016 were then randomized to double-blind PP3M $(\mathrm{N}=504)$ or PP1M $(\mathrm{N}=512$; Figure $\mathrm{S} 1)$. Of the patients randomized to PP3M, 411 (81.5\%) completed 6 months of follow-up and formed the ITT population and 260 patients (63.3\%) achieved remission. No significant differences in baseline demographic characteristics were observed between patients who achieved remission and those who did not (Table S2).

\section{Univariate analysis}

Comparison of symptom scores at Week 17 (prior to treatment randomization) for patients who did and did not achieve 6-month remission (remission positive and remission negative) on PP3M treatment revealed significant group differences: lower mean PANSS total score and Marder factor scores for positive symptoms, negative symptoms, and disorganized thoughts; lower mean CGI-S total score; and higher mean PSP total score (Table 1). No differences were observed between remission groups on Week 17 PANSS Marder factor scores for uncontrolled hostility/excitement and anxiety/depression.

Univariate logistic regression models identified the following predictors of persistent remission on PP3M: PANSS total score at double-blind baseline; Marder factor scores for positive symptoms, negative symptoms, and disorganized thoughts; CGI-S total score; and PSP total score at doubleblind baseline (Table 2). The predicted probabilities and corresponding $95 \%$ CIs of achieving remission at various double-blind baseline scores for PANSS negative symptom factor, CGI-S, and PSP total scores are shown in Figure 1. The probability of achieving remission is $>63 \%$ when the double-blind CGI-S score is mildly ill or better $(\leq 3)$ and the probability of remission is $>72 \%$ when the PSP total score is $>70$, indicating that when patients are doing well on PP1M (minimal symptoms or better), they are more likely to achieve remission on PP3M.

\section{Multiple regression analysis}

In the stepwise multiple regression analysis, the variables that remained significant predictors of remission on PP3M 
Table I Outcome measures at Week 17 of PPIM treatment based on 6-month remission status to PP3M

\begin{tabular}{|c|c|c|c|}
\hline Outcome measure $^{a}$ & Remission positive $(n=260)$ & Remission negative $(n=|5|)$ & $P$-value \\
\hline PANSS total score & $\begin{array}{l}55.2(8.6) \\
56.0(31.0,69.0)\end{array}$ & $\begin{array}{l}61.0(7.0) \\
63.0(32.0,69.0)\end{array}$ & $<0.001$ \\
\hline Positive symptom factor & $\begin{array}{l}\text { I5.2(3.5) } \\
\text { I5.0 (8.0, 28.0) }\end{array}$ & $\begin{array}{l}\text { I6.4(3.6) } \\
16.0(8.0,28.0)\end{array}$ & 0.001 \\
\hline Negative symptom factor & $\begin{array}{l}\text { I5.I (3.5) } \\
\text { I5.0 (7.0, 22.0) }\end{array}$ & $\begin{array}{l}\text { I } 8.3(4.2) \\
\text { | } 9.0(7.0,28.0)\end{array}$ & $<0.001$ \\
\hline Disorganized thoughts factor & $\begin{array}{l}\text { I3.6 (3.I) } \\
\text { I3.0(7.0, 2I.0) }\end{array}$ & $\begin{array}{l}\text { I5.4 (3.I) } \\
\text { I } 6.0(8.0,23.0)\end{array}$ & $<0.001$ \\
\hline Uncontrolled hostility/excitement factor & $\begin{array}{l}5.2(1.6) \\
5.0(4.0,14.0)\end{array}$ & $\begin{array}{l}5.0(1.7) \\
4.0(4.0,13.0)\end{array}$ & 0.25 \\
\hline Anxiety/depression factor & $\begin{array}{l}6.1(2.0) \\
6.0(4.0,12.0)\end{array}$ & $\begin{array}{l}5.8(2.0) \\
5.0(4.0,12.0)\end{array}$ & 0.17 \\
\hline CGI-S total score & $\begin{array}{l}2.8(0.6) \\
3.0(1.0,4.0)\end{array}$ & $\begin{array}{l}3.2(0.6) \\
3.0(2.0,4.0)\end{array}$ & $<0.001$ \\
\hline PSP total score & $\begin{array}{l}68.4(9.3) \\
70.0(40.0,95.0)\end{array}$ & $\begin{array}{l}60.6(10.1) \\
62.0(31.0,80.0)\end{array}$ & $<0.001$ \\
\hline
\end{tabular}

Note: ${ }^{\vee}$ alues are mean (SD) and median (range) at Week 17.

Abbreviations: CGI-S, Clinical Global Impression-Severity; PANSS, Positive and Negative Syndrome Scale; PPIM, paliperidone palmitate I-month formulation; PP3M, paliperidone palmitate 3-month formulation; PSP, Personal and Social Performance.

were as follows: Marder negative symptom factor score at double-blind baseline, CGI-S total score at double-blind baseline, and PSP total score at double-blind baseline (Table 3; Figure 2). A 1-point reduction in the PANSS Marder negative symptom factor score at Week 17 was associated with a $20 \%$ increase in the odds of achieving remission after PP3M treatment. A 1-point reduction in the CGI-S score at Week 17 more than doubled the odds of achieving remission after treatment with PP3M. In this multiple regression analysis, the odds of remission were increased by $42 \%$ in patients achieving a 7-point improvement on the PSP and by $65 \%$ in patients demonstrating a 10-point improvement on the PSP compared to open-label baseline (Table 3).

\section{Discussion}

As treatments have advanced, remission has become a more realistic goal for many individuals with schizophrenia., ${ }^{713}$ Patients who achieve and maintain remission are clinically stable, with absent, borderline, or mild symptoms, ${ }^{7}$ and can therefore devote more time to psychosocial and cognitive rehabilitation. ${ }^{26,27}$ Although symptomatic remission criteria are by definition focused on symptoms, significant positive correlations have been observed between achieving remission and improvements in functional outcomes and quality of life. ${ }^{10}$ Ultimately, many patients with sustained remission who have access to comprehensive well-orchestrated rehabilitation programs may gain empowerment, maintain

Table 2 Univariate logistic regression model of predictors of 6-month remission

\begin{tabular}{|c|c|c|c|}
\hline Predictors & Wald chi-square & $P$-value & OR estimate $(95 \% \mathrm{Cl})$ \\
\hline Age, years & 0.03 & 0.86 & $1.00(0.98,1.10)$ \\
\hline Sex, male vs female & 0.04 & 0.84 & $\mathrm{I} .04(0.70,1.56)$ \\
\hline \multicolumn{4}{|l|}{ Race } \\
\hline Black or African vs White & 1.08 & 0.30 & $\mathrm{I} .74(0.6 \mathrm{I}, 4.94)$ \\
\hline Others vs White & 0.12 & 0.73 & $0.93(0.61,1.42)$ \\
\hline Duration of illness since first diagnosis, years & 0.20 & 0.65 & $1.00(0.97,1.02)$ \\
\hline \multicolumn{4}{|l|}{ Outcome measures at Week 17} \\
\hline PANSS total score & 40.42 & $<0.001$ & $1.10(1.07,1.14)$ \\
\hline Positive symptom factor & 10.03 & 0.002 & $1.10(1.04,1.16)$ \\
\hline Negative symptom factor & 52.62 & $<0.001$ & $1.27(1.19,1.36)$ \\
\hline Disorganized thoughts factor & 30.04 & $<0.001$ & $1.21(1.13,1.30)$ \\
\hline Uncontrolled hostility/excitement factor & 1.30 & 0.25 & $1.08(0.95,1.23)$ \\
\hline Anxiety/depression factor & 1.90 & 0.17 & $1.07(0.97,1.19)$ \\
\hline CGI-S total score & 35.64 & $<0.001$ & $3.55(2.34,5.38)$ \\
\hline PSP total score & 46.84 & $<0.001$ & $1.09(1.06,1.11)$ \\
\hline
\end{tabular}

Abbreviations: CGI-S, Clinical Global Impression-Severity; PANSS, Positive and Negative Syndrome Scale; PSP, Personal and Social Performance. 
A

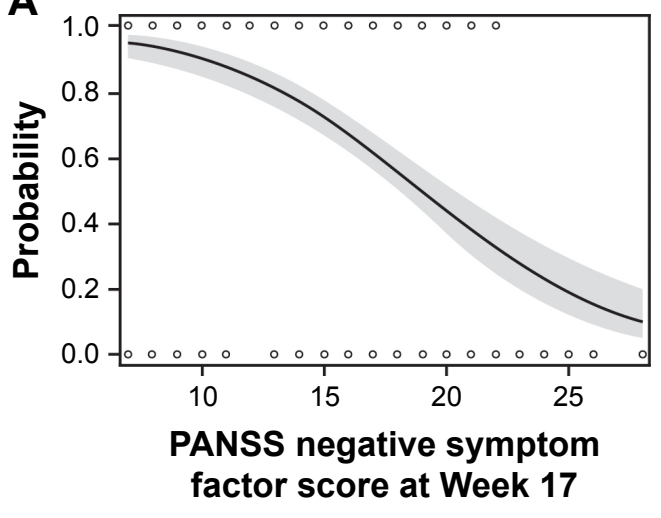

B

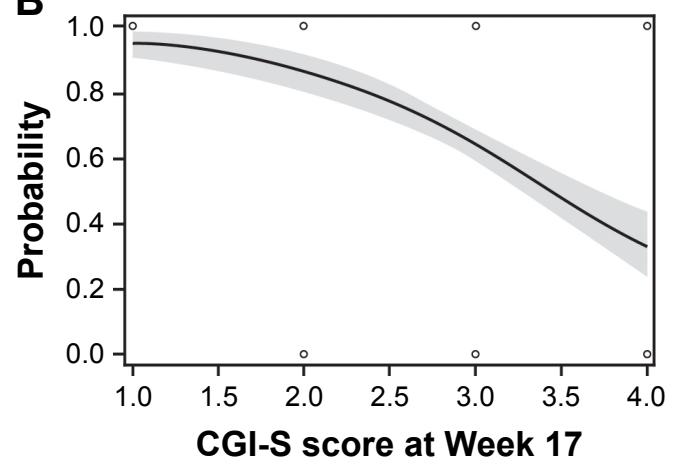

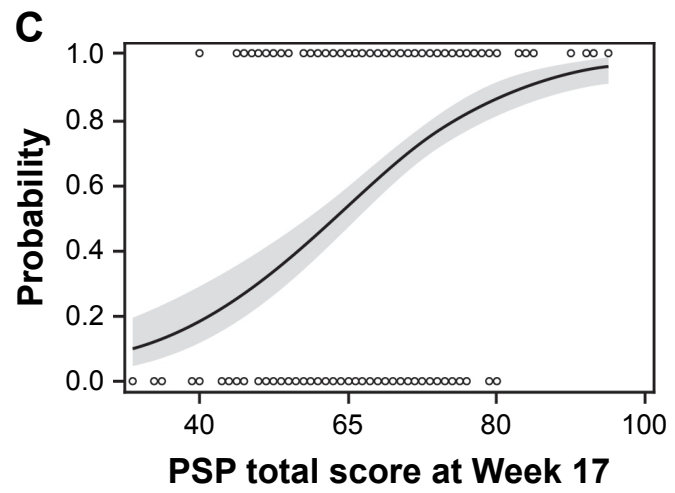

Figure I Predicted probabilities of remission using the univariate model for each significant predictor of remission: (A) PANSS Marder negative symptom factor, (B) CGI-S, and (C) PSP total score. ${ }^{a}$

Notes: ${ }^{2}$ Solid lines with $95 \% \mathrm{Cl}$ bands show predicted probability of remission. Markers at $\mathrm{Y}=0$ (no remission) and $\mathrm{Y=I}$ (remission) list actual observations.

Abbreviations: CGI-S, Clinical Global Impression-Severity; PANSS, Positive and Negative Syndrome Scale; PSP, Personal and Social Performance.

satisfying interpersonal relationships, and be able to achieve greater functional independence on the road to recovery.

In this post hoc analysis of data from the Phase III noninferiority study of PP3M, 63.3\% of the eligible patients (who were required to remain in the double-blind treatment phase $\geq 6$ months) achieved 6 months of symptomatic remission during double-blind treatment with PP3M. In another analysis of this trial, ${ }^{24} 50.3 \%$ of the patients receiving PP3M remained in symptomatic remission and $42.5 \%$ achieved functional remission (assessed using PSP scores) during the final 6 months of the study. In the previous analysis, all patients were included if they received $\geq 1$ dose of PP3M during the double-blind period, regardless of how long they remained in the study; any patient who did not stay in the study $\geq 6$ months was automatically counted as not in remission (symptomatic or functional).

While remission was not a primary endpoint in the study, the current analysis is intended to provide guidance on factors during PP1M treatment that are predictive of successful treatment after transition to PP3M. The term "adequately treated" refers to patients who met stabilization criteria during the transition phase. We were able to identify PANSS Marder negative symptom factor score, PSP improvement, and CGI-S severity as predictive of achieving sustained 6-month remission on PP3M. CGI-S severity had the greatest effect, with a 1-point difference in severity more than doubling the

Table 3 Multivariate logistic regression model of predictors of 6-month remission

\begin{tabular}{|c|c|c|c|}
\hline Predictors & Wald chi-square & $P$-value & OR estimate $(95 \% \mathrm{Cl})$ \\
\hline PANSS negative symptom factor at Week 17 & 27.40 & $<0.001$ & $1.20(1.12,1.29)$ \\
\hline CGI-S total score at Week 17 & 11.03 & $<0.001$ & $2.21(1.38,3.52)$ \\
\hline PSP total score at Week 17 & 13.78 & $<0.001$ & \\
\hline I-point shift & & & $1.05(1.02,1.08)$ \\
\hline 7-point shift & & & $1.42(I .|8| .7 I)$, \\
\hline 10-point shift & & & $1.65(1.27,2.16)$ \\
\hline
\end{tabular}

Abbreviations: CGI-S, Clinical Global Impression-Severity; PANSS, Positive and Negative Syndrome Scale; PSP, Personal and Social Performance. 


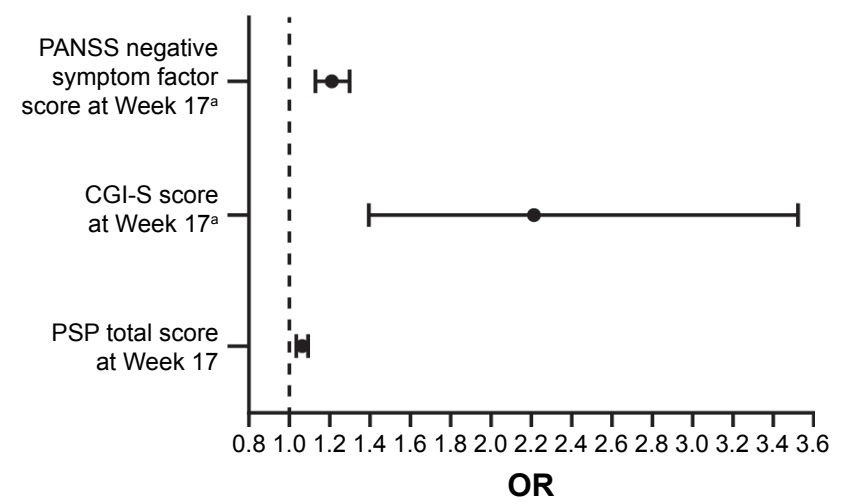

Figure 2 Variables predicting achievement of remission with PP3M during the double-blind treatment phase (multivariate analyses).

Notes: aScoring direction is reversed for the variable. OR indicates that I-point decrease in the scale score increases odds of achieving remission by corresponding unit in $\mathrm{x}$-axis.

Abbreviations: CGI-S, Clinical Global Impression-Severity; PANSS, Positive and Negative Syndrome Scale; PP3M, paliperidone palmitate 3-month formulation; PSP, Personal and Social Performance.

odds of remission. Because a 1-point difference in CGI-S severity is considered detectable by clinicians, our findings suggest that patients with clearly observable benefit on PP1M monotherapy, received every 4 weeks, are among the most suitable candidates for potential transition to PP3M. With regard to the practical applications of the current findings, the CGI-S is the easiest and most commonly used measure in clinical practice. Although improvement on the PSP was also associated with success on PP3M treatment, the PSP is not commonly used in the clinic. However, asking a few specific questions during patient interviews may potentially capture similar information as collected by the PSP. Strong correlations have been demonstrated between the total PSP score and its Work and Socially Useful Activities subscale. ${ }^{28}$ Therefore, asking routine questions such as "Is the patient working? ...going to school? ... doing household chores? ... participating in hobbies?" may be helpful in guiding treatment decisions.

Treatment with PP3M represents a different paradigm in the management of schizophrenia, given the extended half-life and resulting dosing intervals. ${ }^{23}$ Benefits associated with PP3M include the requirement for only 4 injections per year; the longer half-life of PP3M that provides effective paliperidone plasma levels for an even longer duration, allowing medication exposure beyond the scheduled injection interval; and a longer period of symptomatic stability after drug discontinuation in the event of scheduling challenges ${ }^{19}$ or patients who are temporarily unwilling to continue treatment. Patients may still be seen on a monthly basis for clinical assessment, which can focus on areas beyond psychotropic medication and therapeutic interactions, but the clinical consequences of missing such visits would be diminished.

\section{Limitations}

This work has several limitations that affect interpretation of the results. The variables tested in this analysis and its overall findings are based on data from a single, controlled, clinical trial database with an explanatory design that included multiple inclusion and exclusion criteria. Therefore, the results may not be generalizable to broader patient groups. Furthermore, the participants were not selected randomly (eg, in the double-blind treatment period, participants were an enriched population that both responded to and tolerated PP1M through the US label recommendations of adequate treatment) and were treated in the context of a highly supervised clinical trial that may not reflect results in a naturalistic setting. Both design considerations increase uncertainty about the generalizability of the results. The predictor variables tested for significance in achieving remission were limited to those collected as part of the clinical trial; thus, it is possible that other important predictors of remission were not evaluated.

\section{Conclusion}

For clinicians, the key study finding may be that patients who demonstrate global improvement on the CGI-S during PP1M treatment, administered as recommended every 4 weeks without supplementation, are likely to achieve remission after switching to PP3M. In conclusion, patients who demonstrate early clinically meaningful improvements in disease symptoms and severity while establishing a stable PP1M dosage are more likely to achieve remission after transition to PP3M.

\section{Abbreviations}

CGI-S, Clinical Global Impression-Severity; DSM-IV, Diagnostic and Statistical Manual of Mental Disorders, Fourth Edition; ITT, intent-to-treat; LAI, long-acting injectable; PANSS, Positive and Negative Syndrome Scale; PP1M, paliperidone palmitate 1-month formulation; PP3M, paliperidone palmitate 3-month formulation; PSP, Personal and Social Performance; RSWG, Remission in Schizophrenia Working Group.

\section{Acknowledgments}

Writing and editorial support for the development of this manuscript were provided by Wilson Joe, PhD, of MedErgy, and funded by Janssen Scientific Affairs, LLC. The sponsor was involved in the study design; the collection, analysis, and interpretation of the data; the writing of the report; and the decision to submit the article for publication. These data have been previously presented, in part, in a poster by Nash AI, et al. at the 30th Annual Psych Congress (September 16-19, 2017; New Orleans, LA; https://www.psychcongress. 
com/posters/predictors-achieving-remission-schizophreniapatients-treated-paliperidone-palmitate-3).

\section{Author contributions}

Nash and Kim contributed to the study conception and design and the development of the manuscript. Turkoz, Savitz, and Mathews contributed to the study conception and design, the data acquisition and interpretation, and the development of the manuscript. All authors contributed to data analysis, drafting and revising the article, gave final approval of the version to be published, and agree to be accountable for all aspects of the work.

\section{Disclosure}

Nash, Turkoz, Savitz, Mathews, and Kim are employees of Janssen (a Johnson \& Johnson company) and hold stock in Johnson \& Johnson. The authors report no other conflicts of interest in this work.

\section{References}

1. Lieberman JA, Perkins D, Belger A, et al. The early stages of schizophrenia: speculations on pathogenesis, pathophysiology, and therapeutic approaches. Biol Psychiatry. 2001;50(11):884-897.

2. Millier A, Schmidt U, Angermeyer MC, et al. Humanistic burden in schizophrenia: a literature review. J Psychiatr Res. 2014;54:85-93.

3. Jin H, Mosweu I. The societal cost of schizophrenia: a systematic review. Pharmacoeconomics. 2017;35(1):25-42.

4. Cloutier M, Aigbogun MS, Guerin A, et al. The economic burden of schizophrenia in the United States in 2013. J Clin Psychiatry. 2016; 77(6):764-771.

5. Hsiao CY, Lee CT, Lu HL, Tsai YF. Living with schizophrenia: healthrelated quality of life among primary family caregivers. $J$ Clin Nurs. 2017;26(23-24):5151-5159.

6. Awad AG, Voruganti LNP. The burden of schizophrenia on caregivers: a review. Pharmacoeconomics. 2008;26(2):149-162.

7. Andreasen NC, Carpenter WT, Kane JM, Lasser RA, Marder SR, Weinberger DR. Remission in schizophrenia: proposed criteria and rationale for consensus. Am J Psychiatry. 2005;162(3):441-449.

8. Alaqeel B, Margolese HC. Remission in schizophrenia: critical and systematic review. Harv Rev Psychiatry. 2012;20(6):281-297.

9. Docherty JP, Bossie CA, Lachaux B, et al. Patient-based and clinicianbased support for the remission criteria in schizophrenia. Int Clin Psychopharmacol. 2007;22(1):51-55.

10. Lambert M, Karow A, Leucht S, Schimmelmann BG, Naber D. Remission in schizophrenia: validity, frequency, predictors, and patients' perspective 5 years later. Dialogues Clin Neurosci. 2010;12(3):393-407.

11. Brissos S, Dias VV, Balanzá-Martinez V, Carita AI, Figueira ML. Symptomatic remission in schizophrenia patients: relationship with social functioning, quality of life, and neurocognitive performance. Schizophr Res. 2011;129(2-3):133-136.
12. Olivares JM, Sermon J, Hemels M, Schreiner A. Definitions and drivers of relapse in patients with schizophrenia: a systematic literature review. Ann Gen Psychiatry. 2013;12(1):32.

13. Kane JM. Treatment strategies to prevent relapse and encourage remission. J Clin Psychiatry. 2007;68(Suppl 14):27-30.

14. Pennington M, Mccrone P. The cost of relapse in schizophrenia. Pharmacoeconomics. 2017;35(9):921-936.

15. Lieberman JA, Stroup TS, Mcevoy JP, et al. Effectiveness of antipsychotic drugs in patients with chronic schizophrenia. $N$ Engl J Med. 2005;353(12):1209-1223.

16. Cooper D, Moisan J, Grégoire JP. Adherence to atypical antipsychotic treatment among newly treated patients: a population-based study in schizophrenia. J Clin Psychiatry. 2007;68(6):818-825.

17. Moisan J, Grégoire JP. Patterns of discontinuation of atypical antipsychotics in the province of Québec: a retrospective prescription claims database analysis. Clin Ther. 2010;32(Suppl 1):S21-S31.

18. Bossie CA, Alphs LD, Correll CU. Long-acting injectable versus daily oral antipsychotic treatment trials in schizophrenia: pragmatic versus explanatory study designs. Int Clin Psychopharmacol. 2015;30(5): 272-281.

19. Weiden PJ, Kim E, Bermak J, Turkoz I, Gopal S, Berwaerts J. Does halflife matter after antipsychotic discontinuation? A relapse comparison in schizophrenia with 3 different formulations of paliperidone. J Clin Psychiatry. 2017;78(7):e813-e820.

20. Marcus SC, Zummo J, Pettit AR, Stoddard J, Doshi JA. Antipsychotic adherence and rehospitalization in schizophrenia patients receiving oral versus long-acting injectable antipsychotics following hospital discharge. J Manag Care Spec Pharm. 2015;21(9):754-768.

21. INVEGA TRINZA (paliperidone palmitate) prescribing information, Titusville, NJ: Janssen Pharmaceutica, Inc; 2017.

22. Berwaerts J, Liu Y, Gopal S, et al. Efficacy and safety of the 3-month formulation of paliperidone palmitate vs placebo for relapse prevention of schizophrenia: a randomized clinical trial. JAMA Psychiatry. 2015;72(8):830-839.

23. Savitz AJ, Xu H, Gopal S, et al. Efficacy and safety of paliperidone palmitate 3-month formulation for patients with schizophrenia: a randomized, multicenter, double-blind, noninferiority study. Int $J$ Neuropsychopharmacol. 2016;19(7):pyw018.

24. Savitz AJ, Xu H, Gopal S, Nuamah I, Hough D, Mathews M. Paliperidone palmitate 3-month treatment results in symptomatic remission in patients with schizophrenia: a randomized, multicenter, double-blind, and noninferiority study. Int Clin Psychopharmacol. 2017;32(6): 329-336.

25. Marder SR, Davis JM, Chouinard G. The effects of risperidone on the five dimensions of schizophrenia derived by factor analysis: combined results of the North American trials. J Clin Psychiatry. 1997; 58(12):538-546.

26. Liberman RP, Kopelowicz A, Ventura J, Gutkind D. Operational criteria and factors related to recovery from schizophrenia. Int Rev Psychiatry. 2002;14(4):256-272.

27. Velligan DI, Kern RS, Gold JM. Cognitive rehabilitation for schizophrenia and the putative role of motivation and expectancies. Schizophr Bull. 2006;32(3):474-485

28. Turola MC, Comellini G, Galuppi A, Nanni MG, Carantoni E, Scapoli C. Schizophrenia in real life: courses, symptoms and functioning in an Italian population. Int J Ment Health Syst. 2012;6(1):22.
Neuropsychiatric Disease and Treatment

\section{Publish your work in this journal}

Neuropsychiatric Disease and Treatment is an international, peerreviewed journal of clinical therapeutics and pharmacology focusing on concise rapid reporting of clinical or pre-clinical studies on a range of neuropsychiatric and neurological disorders. This journal is indexed on PubMed Central, the 'PsycINFO' database and CAS,

\section{Dovepress}

and is the official journal of The International Neuropsychiatric Association (INA). The manuscript management system is completely online and includes a very quick and fair peer-review system, which is all easy to use. Visit http://www.dovepress.com/testimonials.php to read real quotes from published authors. 